EPJ manuscript No.

(will be inserted by the editor)

\title{
Quantitative Tube Model for Semiflexible Polymer Solutions
}

\author{
Hauke Hinsch $^{1}$, Jan Wilhelm ${ }^{1}$, and Erwin Frey ${ }^{1}$ \\ Arnold Sommerfeld Center for Theoretical Physics and Center of NanoScience, \\ Department of Physics, Ludwig-Maximilians-Universität München, \\ Theresienstrasse 37, D-80333 München, Germany
}

Received: date / Revised version: date

\begin{abstract}
We develop a analytical and quantitative theory of the tube model concept for entangled networks of semiflexible polymers. The absolute value of the tube diameter $L_{\perp}$ is derived as a function of the polymers' persistence length $l_{\mathrm{p}}$ and mesh size $\xi$ of the network. To leading order we find $L_{\perp}=0.32 \xi^{6 / 5} l_{\mathrm{p}}^{-1 / 5}$, which is consistent with known asymptotic scaling laws. Additionally, our theory provides corrections to scaling that account for finite polymer length effects and are dominated by the mesh size to polymer length ratio. We support our analytical studies by extensive computer simulations. These allow to verify assumptions essential to our theoretical description and provide an excellent agreement with the analytically calculated tube diameter. Furthermore, we present simulation data for the distribution function of tube widths in the network.
\end{abstract}

PACS. 83.10.Kn Reptation and tube theories - 87.15.Aa Theory and modeling; computer simulation 87.16.Ka Filaments, microtubules, their networks, and supramolecular assemblies

\section{Introduction}

Filamentous actin (F-actin) is a semiflexible biopolymer that has been the object of intensive research from several domains. As a major constituent of the cytoskeleton, F-actin networks play a key role in the ongoing puzzle of cell mechanics [1,2] and cell motility [3. Depending on the presence of binding proteins, F-actin strands at medium ' concentration can form both chemical (cross-linked) and physical (entangled) networks with different elastic properties [4,5,6]. Besides rheological methods, single polymer visualizations also are feasible, as the strands exceed most synthetic polymers by length. This facilitated the observation of tube-like regions along which DNA or F-actin filaments reptate 7,8 . The confinement of polymers to these cylindrical cages confirmed the "tube model" postulated earlier by de Gennes [9] and Doi and Edwards [10]. This long standing paradigm had proven a successful concept to reduce the complex structure of entangled networks to a single polymer problem.

In such entangled networks, polymers can effortlessly slide past each other but are not allowed to cross. Their interaction is thus mainly of entropic nature as entanglements mutually restrict the accessible configuration space. Grasping this feature in a single polymer model has lead to the famous tube model [9, 10. The suppression of transverse undulations of a test polymer by the surrounding polymers (Fig 1) is modelled by a tube. This tube follows

Send offprint requests to: frey@lmu.de

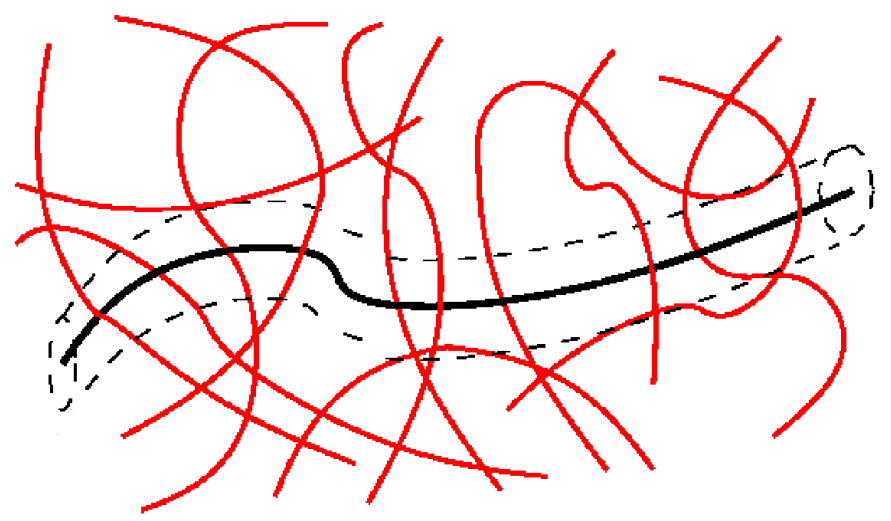

Fig. 1. The effect of all surrounding polymers that hinder the test polymer's transverse displacement is described by a hypothetical tube.

the average path of the test polymer and its profile is frequently modelled by a harmonic potential. The average strength of this potential is determined by the local density of the network. The tube concept has proven a successful tool to derive scaling laws for several network properties [10,11. For example, due to the confinement energy of the filament inside, the tube diameter can be connected to mechanical properties of the network, e.g. the different moduli 6, 12, 13. However, due to the phenomenological nature of the tube model, most of its benefits have been mainly qualitative. Recently, also quantitative predictions of the plateau modulus and the tube diameter of flexible 
Hauke Hinsch et al.: Quantitative Tube Model for Semiflexible Polymer Solutions

polymers melts were achieved by a novel approach based on the microscopic foundations and the topological structure of the network [14,15].

Even if most concepts developed for flexible polymers can not be carried over to the semiflexible case with its large persistence length, the tube model is perfectly applicable as well. However, while in general scaling laws of the tube diameter [16] or the plateau modulus [13] are well established, quantitative theories are still under debate and lack from approval by measurements of sufficient accuracy. Again the challenge is to make the successful tube model quantitative by connecting the phenomenological tube and its microscopic origins. In the present work, we contribute to the discussion by supplying an absolute value for the tube diameter from a theory supported by extensive computer simulations.

We will proceed as follows: in Section 2 the model under investigation is defined and all relevant length scales are discussed. By the analysis of the free energy cost for confining a polymer to a hypothetical tube, the tube diameter is derived as a function of Odijk's deflection length for finite-length polymers. The appropriate deflection length for a given polymer concentration and persistence length is derived in the following sections. To this end, the polymer is modeled by a sequence of independent rods in Section 3. Criteria for the correct choice of the independent rod length and a self-consistent determination of the tube diameter are developed, before a final result for the tube diameter is obtained in Section 4. Extensive numerical simulations supporting these result and providing additional insight are presented in Section 5 followed by our conclusion in Section 6 .

\section{Model Definition}

We consider a mono-disperse network of physically entangled polymers with a particular focus on pure solutions of the biopolymer F-actin. The polymer density is given by the number $\nu$ of polymers of length $L$ per unit volume. The polymers are of bending stiffness $\kappa$ corresponding to a persistence length $l_{\mathrm{p}}=\kappa / k_{\mathrm{B}} T$. A single polymer's configuration $\mathbf{r}(s)$ is parameterized by the arc length $s$ and the average distance between the polymer chains can be characterized by a mesh size $\xi:=\sqrt{3 /(\nu L)}$ 1. We will describe the constituent polymers by the worm-like chain model 17, 18 and exploit the tube model concept 9, 10 to reduce the description of the network to a single polymer and its neighbors. In the following we will begin our analysis with an investigation of the different length scales involved in the system.

1 The mesh size has the unit length and can be interpreted as an average distance between network constituents. While the denominator $\sqrt{1 / \nu L}$ ensures the correct scaling the numerator is a mere definition.

\subsection{Length Scales}

Typical F-action solutions are polydisperse with a mean filament length $L \approx 22 \mu \mathrm{m}[19$. With a persistence length $l_{\mathrm{p}} \approx 17 \mu \mathrm{m}[20,21$ comparable to its length, it is the textbook example of a semiflexible polymer. At a concentration of $c=0.5 \mathrm{mg} / \mathrm{ml}$ corresponding to $\nu \approx 1 \mu \mathrm{m}^{-3}[22$ the average mesh size equals $\xi \approx 0.4 \mu \mathrm{m}$. We can thus state that the persistence length of a filament is much larger than the distance to its neighbors, $l_{\mathrm{p}} \gg \xi$. Since the tube diameter $L_{\perp}$ is at most of the order of the mesh size, this additionally implies $l_{\mathrm{p}} \gg L_{\perp}$. The polymer will thus not deviate far from the tube center. Consequently, configurations where the polymer folds back onto itself are rendered unlikely. This is a minimal requirement to model the tube by a harmonic potential of strength $\gamma$. The potential has to be seen as a hypothetical tube representing the joint contribution of all surrounding polymers which constrain the transverse undulations of a given polymer (see Fig,1).

The energy of a certain polymer contour $\mathbf{r}(s)$ is the sum of the bending energy of the polymer and its confinement into the harmonic potential and is given in the weakly-bending rod approximation by

$$
H(\gamma, \kappa)=\int_{0}^{L} d s\left[\frac{\kappa}{2}\left(\mathbf{r}_{\perp}^{\prime \prime}(s)\right)^{2}+\frac{\gamma}{2} \mathbf{r}_{\perp}^{2}(s)\right]
$$

Here $\mathbf{r}(s)=\left(s, \mathbf{r}_{\perp}(s)\right)$ is a parameterization in arc-length $s$ and transverse displacement $\mathbf{r}_{\perp}(s)=(y(s), z(s))$ from the tube center. The prime denotes a derivative with respect to $s$. This harmonic approximation to the Hamiltonian of the worm-like chain model is valid as long as $\left|\mathbf{r}_{\perp}^{\prime \prime}\right| \ll$ 1 , i.e. as long as the transverse coordinates of the tube coordinate can be considered to remain single valued.

With the thermal average $\langle\cdot\rangle$ the tube diameter can now be defined as

$$
L_{\perp}:=\frac{1}{L}\left\langle\int_{0}^{L} d s \mathbf{r}_{\perp}^{2}(s)\right\rangle .
$$

So far we have identified two length scales: the length scale of persistence length and the total polymer length describing the properties of one specific polymer, and the length scale of mesh size and the tube diameter describing the properties of the network structure. Additionally we introduce the deflection length $L_{\mathrm{d}}:=(\kappa / \gamma)^{1 / 4}$ as a third useful length scale. It is interpreted below as that length on which interactions between single polymer and network occur. More precisely, it is a measure for the number of contacts of the polymer with the tube walls. For large confinement strength $\gamma$ the tube is small, making interaction with the encaged polymer more likely and therefore resulting in a small deflection length. On the other hand, for a large polymer rigidity $\kappa$ transverse undulations allowing contacts with the tube walls are energetically unfavorable and the distance between contact will decrease. For $l_{\mathrm{p}} \gg L_{\perp}$ we expect the deflection length to be distinctively smaller than the polymer length, but also larger than the tube diameter. For quantification we consider the free energy cost $\Delta F(\gamma)$ of confining the polymer to the tube. It 
can be found from the partition sum that is obtained as a path integral over all polymer configurations:

$$
\exp [-\beta \Delta F(\gamma)]=\int \mathcal{D}\left[\mathbf{r}_{\perp}(s)\right] \exp [-\beta H(\kappa, \gamma)]
$$

with $\beta=1 / k_{\mathrm{B}} T$. In the limit of infinitely long polymers the free energy cost is 23 .

$$
\Delta F=\sqrt{2} k_{\mathrm{B}} T \frac{L}{L_{\mathrm{d}}} .
$$

This result fits into the picture of the deflection length as measure for the average distance between successive collisions of the polymer with its tube. If the typical distance between two collisions is given by $L_{\mathrm{d}}$, the free energy loss results as the sum over all $L / L_{\mathrm{d}}$ points of contact where every collision costs one $k_{\mathrm{B}} T$. The free energy now allows one to derive the tube diameter as

$$
L_{\perp}^{2}=\frac{2}{L} \frac{\partial \Delta F}{\partial \gamma}=\frac{L_{\mathrm{d}}^{3}}{\sqrt{2} l_{\mathrm{p}}} .
$$

In the limit of infinite polymer length we have thus derived the tube diameter as a function of the deflection length by differentiation of the free energy cost.

The above consideration also sets the road map for the remaining work. To calculate the tube diameter for the network, we need first to connect free energy and tube diameter for polymers of finite length and then derive the deflection length for the model under investigation.

\subsection{Finite length Polymers}

For finite size polymers the path integral in Eq.(3) can be evaluated exactly [23,24] and with the dimensionless deflection length $l_{\mathrm{d}}:=L_{\mathrm{d}} / L$ results in

$$
\Delta F=-2 k_{\mathrm{B}} T g\left(l_{\mathrm{d}}\right)
$$

with

$$
g\left(l_{\mathrm{d}}\right)=\ln \left(l_{\mathrm{d}}^{2}\right)-\frac{1}{2} \ln \left(\sinh ^{2} \frac{1}{\sqrt{2} l_{\mathrm{d}}}-\sin ^{2} \frac{1}{\sqrt{2} l_{\mathrm{d}}}\right) .
$$

The limit of small $l_{\mathrm{d}}$ that is guaranteed by $L \gg L_{\mathrm{d}}$ as stated above, allows an expansion

$$
g\left(l_{\mathrm{d}}\right)=-\frac{1}{\sqrt{2} l_{\mathrm{d}}}+\ln \left(l_{\mathrm{d}}^{2}\right)+\mathcal{O}\left(e^{-1 / l_{\mathrm{d}}}\right) \quad \text { for } \quad l_{\mathrm{d}} \rightarrow 0
$$

where the first term is just the result for polymers with infinite length (4). Upon again using the relation $L_{\perp}^{2}=$ $(2 / L)\left(\partial \Delta F\left(l_{d}\right) / \partial \gamma\right)$ with the inner derivative $\partial l_{\mathrm{d}} / \partial \gamma=$ $-\left(L^{4} / 4 \kappa\right) l_{\mathrm{d}}^{5}$ the tube diameter becomes

$$
L_{\perp}^{2}=\frac{L^{3}}{2 l_{\mathrm{p}}} l_{\mathrm{d}}^{5} g^{\prime}\left(l_{\mathrm{d}}\right) .
$$

For later convenience we simplify this to $l_{\perp}^{2}=h\left(l_{\mathrm{d}}\right)$ by introducing a dimensionless tube width $l_{\perp}$ and function $h(x)$ as

$$
l_{\perp}^{2}:=\frac{L_{\perp}^{2} l_{\mathrm{p}}}{L^{3}} \quad \text { and } \quad h(x):=\frac{x^{5} g^{\prime}(x)}{2} .
$$

This relation connects the wanted tube diameter to the deflection length and hence to the hypothetical tube potential $\gamma$ at a given bending rigidity. In the following we will further investigate the tube properties and set up a model that allows one to derive the deflection length and thereby the hypothetical harmonic tube potential strength from the polymer concentration and persistence length.

\section{Independent Rod Model}

For simplification and as an anticipation towards the computer simulations, consider for the time being a polymer in a two-dimensional (2D) plane. In this case the transverse displacement vector $\mathbf{r}_{\perp}$ reduces to a single component. The undulations of the test polymer in $2 \mathrm{D}$ are hindered by point-like obstacles as depicted in Fig. 2 (top). These obstacles represent the cuts of the surrounding polymers in three dimensions with the chosen fluctuation plane. Given an appropriate number of $2 \mathrm{D}$ obstacles equivalent to the density of surrounding polymers in $3 \mathrm{D}$, the transverse displacement will correspond to one of the two components of the displacement vector $\mathbf{r}_{\perp}$, if we assume the fluctuations of these components to be independent. Bearing in mind the large persistence length compared to the mesh size, the surrounding polymers in 3D are modelled as rigid rods and the area density $\rho_{\mathrm{MC}}$ of obstacles in $2 \mathrm{D}$ corresponding to a polymer concentration $\nu$ in the 3D network is $\rho_{\mathrm{MC}}=2 \nu L / \pi$. It is computed in Appendix $\mathrm{A}$ and will be explicitly needed for the comparison with simulation results.

Recalling the Hamiltonian (1), the polymer's free energy has a bending and an entropic contribution. To minimize the free energy it can be favorable to trade in bending energy for a wider tube. Thereby entropy is gained due to a larger available free volume, but the polymer is forced to sacrifice energy to obtain its curvature (see Fig. 2 (top)). This competition defines a characteristic length $\bar{L}$ that has to be of the order of the deflection length $L_{\mathrm{d}}$, since this is the length scale characterizing interaction of the test polymer and its environment.

In the following we will develop an analytical theory based on an independent rod model (IRM) that is inspired by the competition we have just discussed. To this end, we use a simplified model of a semiflexible polymer, in which the flexibility is localized to the joints of a sequence of independent stiff rods of length $\bar{L}$. After deriving the transverse fluctuations of a single independent rod in an environment of fluctuating neighbors, we apply a self-consistency argument to arrive at the corresponding tube width of the full length semiflexible polymer. Note that the analysis is carried out for three dimensions and the 2D simplification only serves for illustration and for simulations later on.

To begin with, consider the test polymer to be divided into independent segments of length $\bar{L}$ that are assumed to be completely rigid rods and are only allowed to undergo transverse fluctuations. As the flexibility in the IRM depends on the number of joints, it is obvious that the choice of $\bar{L}$ is crucial for the resulting tube diameter. Picturing 

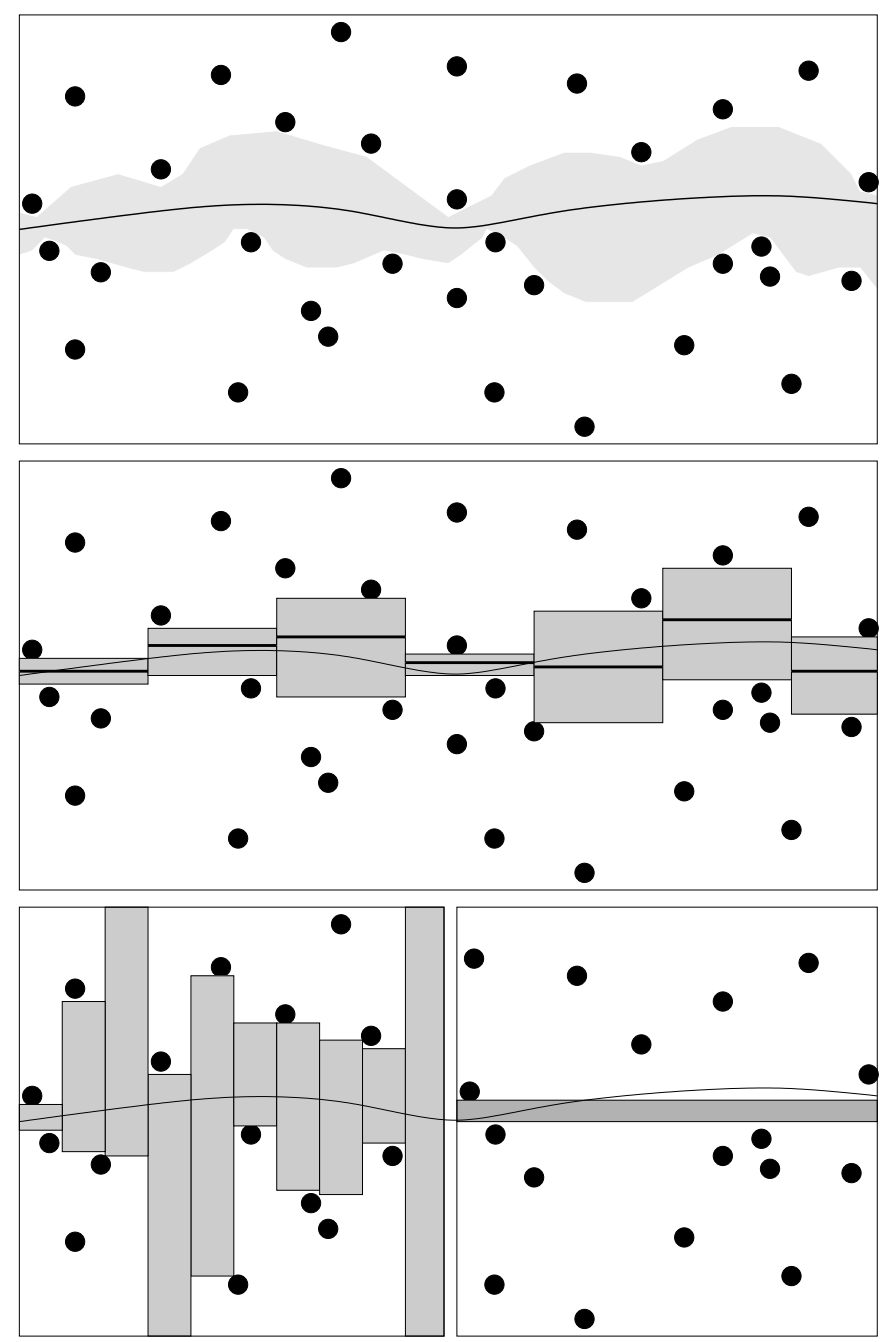

Fig. 2. (top) The fluctuation tube of a semiflexible polymer in a network of constraints is determined by a delicate balance of entropic and bending energy. (middle) Scheme of decomposition of a semiflexible polymer into rigid rods of length $\bar{L}$. The flexibility is localized to the joints between independent rods. Given the proper choice of $\bar{L}$ both models produce the same transverse fluctuation area. (bottom) Small rod length overestimates and large rod length underestimates fluctuations.

the decomposition of the test polymer as in Fig. 2 (middle) it can be seen that the transverse fluctuations of the independent rods are hindered by the two closest obstacle polymers normal to either side of each segment of length $\bar{L}$. If $\bar{L}$ is chosen too large (e.g. $\bar{L}=L$ in the worst case) the area of transverse fluctuations will be much smaller than for a true semiflexible polymer because flexibility is underestimated (Fig. 2 (bottom, right)). On the contrary, if $\bar{L}$ is chosen too small, the normal distance to the nearest obstacle can be quite large (Fig. 2 (bottom, left)). This overestimation of flexibility results in a transverse fluctuation area that is large compared to the polymer we try to model. Before we further discuss the proper choice of $\bar{L}$, we will focus on the behavior of a single independent rod in more detail.
The transverse fluctuation of a single stiff rod in the $(y, z)$-plane are constrained by the projections of the surrounding network constituents to this plane as depicted in Fig. 3 (left). Since the mesh size is much smaller than the persistence length, the surrounding polymers can be assumed to be straight and "dangling ends" are neglected. The size of the shaded cross section will decrease with increasing density of polymers, i.e. with a decreased mesh size. Thus the tube diameter is of order of the mesh size and scales as $L_{\perp} \propto \xi$ for a given 2D plane. Furthermore, an increase of the length $\bar{L}$ of the rigid rod signifies an increase of obstacles that will be projected to the plane. As the average distance between surrounding polymers in direction of the test rod is also given by the mesh size $\xi$, the average number projected onto the plane increases as $\bar{L} / \xi$. As this reduces the cross section area, we finally arrive at an overall scaling of the tube diameter as $L_{\perp} \propto \xi^{2} / \bar{L}$.
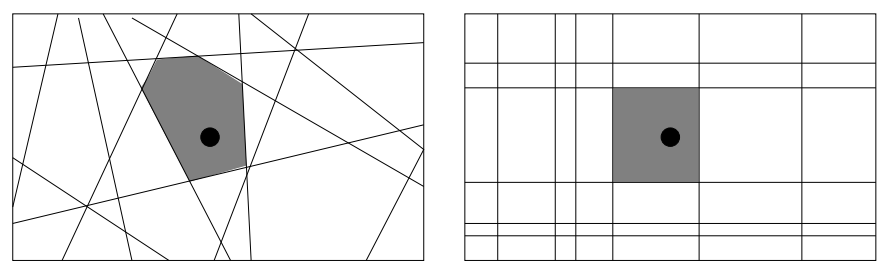

Fig. 3. (left) Projection of constraining polymers to the plane of transverse fluctuations of a test polymer (black dot). As the mesh size is much smaller than the persistence length, the constraining filaments, can be assumed to be straight. The shaded area is the accessible tube area for a specific obstacle configuration. (right) Corresponding setup for a simplified geometry where obstacles can only be aligned with coordinate axes.

Before we quantify this scaling result in the next section, let us first have a closer look at the obstacles. In a self-consistent treatment these evidently have themselves to be regarded as semiflexible polymers of the network and therefore undergo fluctuations around an average position as well. This causes the cross section area to smear out, as the test polymer has now a non-vanishing probability to take on values behind the average obstacle position. In terms of a confinement potential the cross section is no more described by an infinite well, but by some continuous potential which earlier has been assumed to be harmonic with strength $\gamma$ per unit length of a polymer. The obstacle fluctuations will also be modelled as Gaussian and to distinguish between the test polymer mean square displacement $L_{\perp}^{2}$ and the obstacle's, the latter is denoted as $\sigma^{2}$. In a self-consistent treatment of the network the average tube width $L_{\perp}$ of the test polymer is then determined as a function of the obstacle fluctuations $\sigma$, where $\sigma$ is chosen such that $L_{\perp}=\sigma$. Of course, the value $L_{\perp}$ of a single obstacle configuration will not only depend on $\sigma$ but also on the obstacle positions in that specific configuration. Consequently, averaging over all obstacle configurations will result in a distribution $P\left(L_{\perp}\right)$ and self-consistency would then also require a distribution $P(\sigma)$. However, if we assume these distributions to be reasonably peaked, we can use their averages as a good approximation. The 
self-consistency of distributed tube widths is verified by simulations in Section 5 .

\subsection{Single stiff rod in simplified geometry}

According to the assumptions made above the obstacles (in a top view) are completely described by a normal distance $r_{k}$ from the test polymer and an orientation $\alpha_{k}$; compare Fig. 3. We will neglect correlations and assume the obstacles to be uniformly distributed. The probability to find an obstacle with a certain direction at a specified point is independent of the direction and that point. This corresponds to a complete factorization of the network distribution function into single polymer distribution functions.

Consider first a simplified geometry in which all obstacles are either parallel to the $y$ or the $z$ axis as depicted in Fig. 3 (right). As fluctuations in both coordinates are assumed to be independent and equivalent, the task of computing the tube width is reduced to a one dimensional problem with a single coordinate $r$. The network density or mesh size enters as the number $\rho$ of obstacles per unit length. This density should be chosen such, that the average number of obstacles at a certain distance $r$ from the test rod in the IRM is the same as the average number of obstacle polymers featuring a minimal distance $r$ from the test polymer. This density is proportional to the length $\bar{L}$ of the stiff segment and the number of surrounding polymers in a unit volume $\nu L$. The exact relation $\rho=(\pi / 2)(\nu L \bar{L} / 4)$ is calculated in Appendix B.

As the obstacles are assumed to undergo Gaussian fluctuations around their average position $r_{k}$, the corresponding probability density is

$$
P_{0}\left(r-r_{k}, \sigma\right):=\left(2 \pi \sigma^{2}\right)^{-1 / 2} e^{\frac{-\left(r-r_{k}\right)^{2}}{2 \sigma^{2}}} .
$$

If the test rod interacts with only a single obstacle, we can state that the probability to find the test rod at a certain position is given by the fraction of realizations still accessible to the obstacle. In this case

$$
P_{+}\left(r, r_{k}, \sigma\right)=\int_{r}^{\infty} d r^{\prime} P_{0}\left(r^{\prime}-r_{k}, \sigma\right)
$$

is the fraction of configuration space still accessible to the obstacle if the test rod is placed at $r$ (for $r_{k}>0$ ). Completing the integral yields

$$
P_{+}\left(r, r_{k}, \sigma\right)=\frac{1}{2} \operatorname{erfc}\left(\frac{r-r_{k}}{\sqrt{2} \sigma}\right)
$$

and the corresponding probability for obstacles at negative positions $P_{-}\left(r, r_{k}, \sigma\right)$ is simply obtained by a inverted sign of the argument. The probability to find the test rod at a position $r$ for a given configuration of obstacles $\left\{r_{k}\right\}$ is then given by the product of all probabilities

$$
P\left(r,\left\{r_{k}\right\}, \sigma\right)=\frac{1}{N} \prod_{k, r_{k}>0} P_{+}\left(r, r_{k}, \sigma\right) \prod_{k, r_{k}<0} P_{-}\left(r, r_{k}, \sigma\right)
$$

The normalization $N=N\left(\left\{r_{k}\right\}, \sigma\right)$ is determined by the condition $\int d r P\left(r,\left\{r_{k}\right\}, \sigma\right)=1$ and depends on the obstacle configuration.

As the function $P_{+}\left(r, r_{k}, \sigma\right)$ reduces to a Heaviside function in the case of $\sigma \rightarrow 0$, the product in Eq. (14) can be written as $\theta\left(r-r_{-}\right) \theta\left(r-r_{+}\right) /\left(r_{+}-r_{-}\right)$where $r_{+}$and $r_{-}$are the positions of the two closest obstacles. This reduction is justified because all further obstacles are completely shadowed by the two nearest neighbors. In the case of a non-vanishing $\sigma$ the probability distribution $P\left(r,\left\{r_{k}, \alpha_{k}\right\}, \sigma\right)$ will not be rectangular anymore but smear out. The test rod has a non-vanishing probability to be found behind the average position of the closest obstacle and thus a chance to feel the interaction of further network constituents. However, sketching the distribution in Fig. 4, it becomes intuitively clear that this probability rapidly approaches zero for far obstacles or small fluctuation amplitudes $\sigma$. We will exploit this fact in the numerical analysis below and in the simulations.

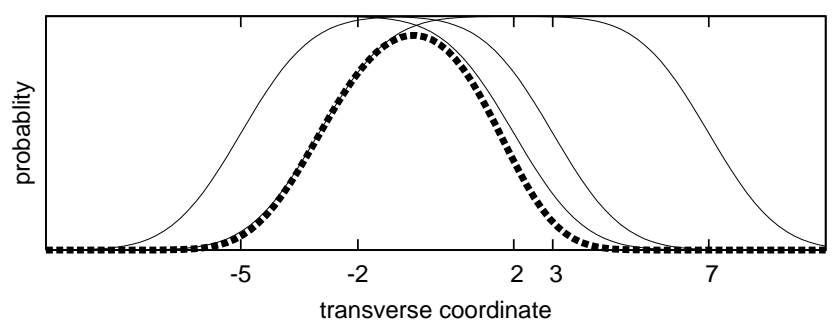

Fig. 4. Probability density to find the test rod at a spatial position for mutual interaction with a single obstacle (solid lines) and resulting probability in an environment of all obstacles (dashed line). The $\mathrm{x}$-axis tics mark the center position of each obstacle. Distant obstacle only have a negligible influence on the overall probability function.

The distribution function, Eq. (14), for the test rod at hand, averages of any function $f(r)$ can now be calculated for a single realization of obstacles as

$$
\overline{f(r)}_{\left\{r_{k}\right\}}=\int d r f(r) P\left(r,\left\{r_{k}\right\}, \sigma\right),
$$

where the index $\left\{r_{k}\right\}$ denotes the specific obstacle configuration. The tube center of the test rod is then

$$
\bar{r}\left(\left\{r_{k}\right\}, \sigma\right):=\bar{r}_{\left\{r_{k}\right\}}
$$

and the width of the probability distribution is the wanted tube diameter

$$
L_{\perp}^{2}\left(\left\{r_{k}\right\}, \sigma\right):=\bar{r}_{\left\{r_{k}\right\}}-\bar{r}_{\left\{r_{k}\right\}}^{2} .
$$


The derived tube diameter of the test rod is not only a function of the fluctuation width $\sigma$ but also of the specific obstacle configuration. Consequently, sampling over different obstacle sets will result in a distribution of values for $L_{\perp}$. As mentioned earlier this distribution should be described by a single characteristic value - consistent with the obstacle fluctuations that have also be assumed to be of equal size. Since the obstacles are uniformly distributed, they can be fully described by the density encoded in the average number of obstacles $\rho$ per line. This is achieved by integrating out all obstacle positions and orientations in $L_{\perp}^{2}\left(\left\{r_{k}\right\}, \sigma\right)$ to arrive at the only density dependent $L_{\perp}^{2}(\rho, \sigma)$. We choose a simple average over a large number $N$ of obstacle sets $\left\{r_{k}\right\}$ like

$$
\left\langle f\left(\left\{r_{k}\right\}\right)\right\rangle_{\rho}=\left(\prod_{k=1}^{N} \int_{-R / 2}^{R / 2} \frac{d r_{k}}{R}\right) f\left(\left\{r_{k}\right\}\right),
$$

where $R=N / \rho$. In this nomenclature the average tube diameter 2 is obtained as $L_{\perp}^{2}(\rho, \sigma)=\left\langle L_{\perp}^{2}\left(\left\{r_{k}\right\}, \sigma\right)\right\rangle_{\rho}$.

Self-consistency is now expressed as

$$
L_{\perp}^{2}(\rho, \sigma)=\sigma^{2}
$$

at the point of self-consistency (PSC) $\sigma=\sigma^{*}$. By measuring length in $1 / \rho$ we can rewrite this to a dimensionless master curve $l(\rho \sigma)$ :

$$
L_{\perp}^{2}(\rho, \sigma)=\frac{1}{\rho^{2}} l(\rho \sigma),
$$

since $L_{\perp}, 1 / \rho$ and $\sigma$ are all lengths, The task of finding the self-consistent tube width $L_{\perp}\left(\rho, \sigma^{*}\right)=\sigma^{*}$ translates to finding $l(C)=C^{2}$ where the constant $C=\rho \sigma^{*}$. As soon as this is achieved, the self-consistent tube diameter is available as a function of density $\rho$ only and hence it depends like

$$
L_{\perp}=\frac{C}{\rho}=\frac{2 C}{3 \pi} \frac{\xi^{2}}{\bar{L}}
$$

on the rod length $\bar{L}$ and mesh size $\xi$.

The numerical determination of $C$ is achieved by an integration using a Monte-Carlo procedure. It includes the $N$-fold integrals over the obstacle positions $r_{k}$ from Eq. (14) as well as the integration over the test polymer position $r$ from Eq. (15). As mentioned above, the probability distribution rapidly decreases at distances far from the closest obstacle. Hence, we have restricted the integration range of the $d r$ integration in the Monte-Carlo samples to values $\left[y_{\min }-5 \sigma, y_{\max }+5 \sigma\right]$ where $y_{\min }$ and $y_{\max }$ are the closest obstacle at either side. Furthermore, the fast decrease of the probability distribution renders the contribution of distant obstacles quasi to zero. We can therefore drop all obstacles with $y_{k} \notin\left[y_{\min }-10 \sigma, y_{\max }+10 \sigma\right]$. The

\footnotetext{
2 Of course, one could also image a different characterization of the average tube diameter, e.g. the median or the maximal diameter. We choose the average as the most obvious quantity experimental groups might measure, e.g. in analyzing different fluorescent microscopy images.
}

results are depicted in Fig. [5] and a graphical solution for the PSC constant results in

$$
C \approx 3.64
$$

Special attention should be paid to the behavior at $\rho \sigma=0$. It provides a good test whether the used IRM is adequate and allows for a verification of the numerics. At a finite density as required by the tube concept, $l(0)$ reflects the situation of immobile obstacles with $\sigma=0$. At this point the tube diameter should remain finite and its value should be given by the density of obstacles. From the obstacle statistics and density per unit length $\rho / 4$, the probability to find the first obstacle at position $r_{ \pm}$is known to be $P\left(r_{ \pm}\right)=\exp \left(-r_{ \pm} \rho / 4\right)$. In the case of fixed obstacles the available fluctuation area is $2 L_{\perp}=r_{+}-r_{-}$and the expectation value $4\left\langle L_{\perp}^{2}\right\rangle=\left\langle\left(r_{+}-r_{-}\right)^{2}\right\rangle$ can be computed from the probability density above. Taking care of the normalization one arrives at $L_{\perp}=\sqrt{8} / \rho$. The master function yields $l(0)=\rho^{2} L_{\perp}^{2}=8$, a value in good agreement with the data (circles) in Fig. 5.

\subsection{Generic 2d Geometry}

If the simplification of axis-parallel obstacle polymers is dropped again, the obstacle configuration needs to be specified by a set of radii $\left\{r_{k}\right\}$ and angles $\left\{\alpha_{k}\right\}$. The probability to find the test rod at a position $(y, z)$ for a given configuration of obstacles $\left\{r_{k}, \alpha_{k}\right\}$ in the two-dimensional case as in Fig. 3 (left) is then again given by the product of all probabilities where different angles have to be accounted for:

$P\left(y, z,\left\{r_{k}, \alpha_{k}\right\}, \sigma\right)=\frac{1}{N} \prod_{k} P_{ \pm}\left(y \cos \alpha_{k}+z \sin \alpha_{k}, r_{k}, \sigma\right)$.

The normalization factor $N=N\left(\left\{r_{k}, \alpha_{k}\right\}, \sigma\right)$ is again determined by the condition $\int d y d z P\left(y, z,\left\{r_{k}, \alpha_{k}\right\}, \sigma\right)=1$.

In a single obstacle configuration the tube diameters $L_{\perp y, z}$ in the $y$ and $z$ direction will in general be different. However, in averaging over all configurations isotropy must be recovered to show

$$
L_{\perp}^{2}(\rho, \sigma)=\left\langle L_{\perp y}^{2}\left(\left\{r_{k}, \alpha_{k}\right\}, \sigma\right)\right\rangle_{\rho}=\left\langle L_{\perp z}^{2}\left(\left\{r_{k}, \alpha_{k}\right\}, \sigma\right)\right\rangle_{\rho} .
$$

The average over obstacle configurations at fixed density of uniformly distributed obstacles is performed as

$$
\left\langle f\left(\left\{r_{k}, \alpha_{k}\right\}\right)\right\rangle_{\rho}=\left(\prod_{k=1}^{N} \int_{0}^{R} \frac{d r_{k}}{R} \int_{0}^{2 \pi} \frac{d \alpha_{k}}{2 \pi}\right) f\left(\left\{r_{k}, \alpha_{k}\right\}\right)
$$

with integration range being again $R=N / \rho$. Note that contrary to the simplified geometry the obstacle density per unit length $\rho$ in this case is given by $\rho=(\pi / 2)(\nu L \bar{L})$. Evaluating the integrals in Eq. (25) again by the MonteCarlo method results in the data plotted in Fig. 5 (triangles), where suppression of irrelevant obstacles was implied analog to the simplified geometry. The results do not deviate much from the data obtained earlier (circles), i.e. 


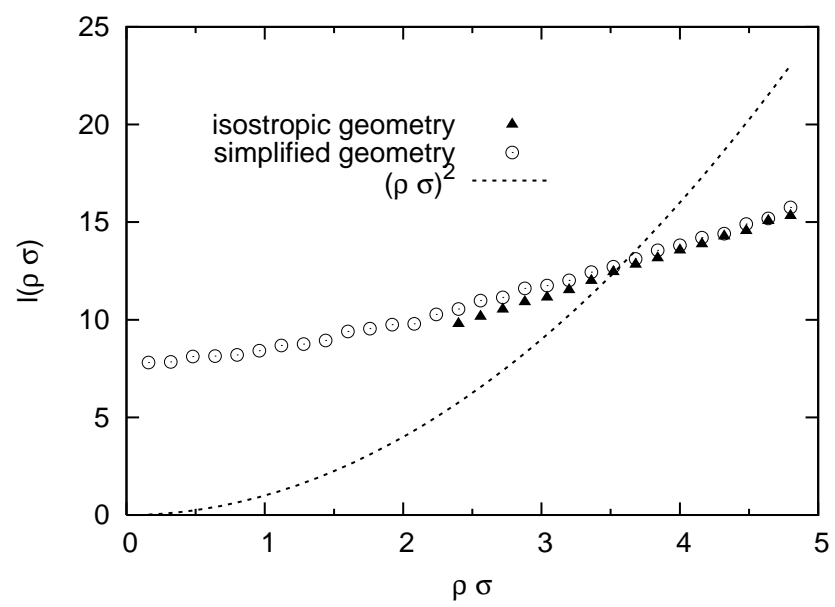

Fig. 5. Master curve $l(\rho \sigma)$ of the tube diameter rescaled by obstacle density obtained by MC simulation for simplified (circles) and generic geometry (triangles); intersection with quadratic obstacle fluctuation amplitude marks the point of self-consistence. The error of the simplified geometry is surprisingly small.

the mistake in using a simplified geometry is surprisingly small. Again the value of the PSC is obtained graphically. It yields

$$
C \approx 3.52
$$

and will be used in the remainder of this work.

\subsection{Choice of Independent Rod Length}

As discussed before and illustrated in Fig. 2 (bottom) the choice of $\bar{L}$ is crucial for the success of the IRM. The number $L / \bar{L}$ of independent rods can be regarded as a measure for the flexibility of the modeled polymer and has to be chosen such that the transverse excursions of the ensemble of stiff rods equal the fluctuations of the actual semiflexible polymer. To this end we consider both systems in a generic harmonic potential

$$
U[y(s)]=\frac{\gamma}{2}\left[y(s)-y^{0}(s)\right]^{2}
$$

with the potential minimum $y^{0}(s)$ as a Gaussian variable with $\left\langle y^{0}(s) y^{0}\left(s^{\prime}\right)\right\rangle=\alpha \delta\left(s-s^{\prime}\right)$. This corresponds to the assumption of a "Gaussian random backbone" as a general property of the tube. We use this intuitive assumption as one possible prerequisite to determine the segment length $\bar{L}$. Of course, other possibilities can be imagined. Note that the simulations in Sec. 5 will justify this assumptions a posteriori.

The average position $\overline{y(s)}$ as a functional of a given potential $y^{0}(s)$ is obtained as an average over all polymer configurations in this potential. Averaging then over all potential conformations yields the the mean square of the polymer's transverse fluctuations $\left\langle\overline{y(s)}^{2}\right\rangle$. The overline thus denotes an average in a given potential and the brackets denote an average over all potentials. While the transverse fluctuations of a rigid rod are a function of the potential parameters $\alpha, \gamma$ only, the response of a semiflexible polymer will additionally depend on its stiffness. This evidently provides a tool to connect the semiflexible polymer persistence length and the length $\bar{L}$ from the IRM by demanding that the fluctuations $\left\langle\overline{y(s)}^{2}\right\rangle$ for given potential parameters $\alpha, \gamma$ are the same for both cases.

Starting with the IRM, it is sufficient to consider only one stiff rod, as the individual rods are statistically independent. The average position is then

$$
\bar{y}=\frac{1}{\bar{L}} \int_{0}^{\bar{L}} \operatorname{ds} y^{0}(s)
$$

and the transverse fluctuations

$$
\left\langle\bar{y}^{2}\right\rangle=\frac{1}{\bar{L}^{2}} \int_{0}^{\bar{L}} d s \int_{0}^{\bar{L}} d s^{\prime}\left\langle y^{0}(s) y^{0}\left(s^{\prime}\right)\right\rangle=\frac{\alpha}{\bar{L}} .
$$

For the semiflexible polymer the fluctuations of polymer and tube potential are decomposed into modes (Appendix C):

$$
\left\langle\bar{y}^{2}\right\rangle=\frac{1}{L} \int_{0}^{L}\left\langle\overline{y(s)}^{2}\right\rangle=\frac{1}{L} \sum_{k}\left\langle\overline{y k}^{2}\right\rangle,
$$

where the mode analysis yields $\overline{y_{k}}=y_{k}^{0} /\left(1+q_{k}^{4} l_{\mathrm{d}}^{4}\right)$ (compare Eq. (510) with $q_{k} \approx \pi(k+1 / 2)$. Using now the correlations of the Gaussian random tube profile and the identity (53) the polymer fluctuations can be related to the deflection length as:

$$
\left\langle\bar{y}^{2}\right\rangle=\frac{1}{L} \sum_{k} \frac{\left\langle\left(y_{k}^{0}\right)^{2}\right\rangle}{\left(1+q_{k}^{4} l_{\mathrm{d}}^{4}\right)^{2}}=\frac{\alpha}{L} \frac{h^{\prime}\left(l_{\mathrm{d}}\right)}{4 l_{\mathrm{d}}^{3}} .
$$

Equating the fluctuations for the IRM and the semiflexible polymer fixes the segment length to

$$
\bar{L}=L \frac{4 l_{\mathrm{d}}^{3}}{h^{\prime}\left(l_{\mathrm{d}}\right)} .
$$

Concluding the last section, we have obtained the tube diameter for a sequence of independent rods of length $\bar{L}$ and derived a condition how to fix this length to correctly mimic the behavior of a semiflexible polymer in a network of same mesh size. It has turned out the the criteria for the correct rod size is a function of the deflection length.

\section{Results}

If we recall that the tube diameter for a semiflexible polymer was derived in Sec. 2 from the Hamiltonian with a likewise dependence on deflection length, we are now equipped to set up an implicit equation to determine this deflection length. Afterwards the tube diameter can be derived from a simple calculation.

Equating the expressions for the tube diameter of the polymer (10) and the IRM (21) respectively yields

$$
L_{\perp}^{2}=\frac{L^{3}}{l_{\mathrm{p}}} h\left(l_{\mathrm{d}}\right)=\frac{4 C^{2}}{9 \pi^{2}} \frac{\xi^{4}}{\bar{L}^{2}} .
$$


With the correct rod length (32) the implicit equation for the dimensionless deflection length is

$$
h\left(l_{\mathrm{d}}\right)=\frac{C^{2}}{32 \pi^{2}} \frac{\left[h^{\prime}\left(l_{\mathrm{d}}\right)\right]^{2}}{l_{\mathrm{d}}^{6}} \frac{l_{\mathrm{p}} \xi^{4}}{L^{5}} .
$$

Solving this equation, determines $l_{\mathrm{d}}$ from the system's parameter $l_{\mathrm{p}}, L$ and $\xi$. It is achieved by introducing a dimensionless function

$$
l_{\mathrm{p}} \xi^{4} / L^{5}=j\left(l_{\mathrm{d}}\right):=\frac{l_{\mathrm{d}}^{6} 32 \pi^{2} h\left(l_{\mathrm{d}}\right)}{C^{2}\left[h^{\prime}\left(l_{\mathrm{d}}\right)\right]^{2}} .
$$

Inversion then yields

$$
l_{\mathrm{d}}=j^{-1}\left(l_{\mathrm{p}} \xi^{4} / L^{5}\right)
$$

With the abbreviation $D=3 C / \pi$ we finally obtain for the dimensional deflection length to first order and second order in the argument of $j^{-1}$ :

$$
L_{\mathrm{d}}=\frac{D^{2 / 5}}{2^{13 / 10}} \xi^{4 / 5} l_{\mathrm{p}}^{1 / 5}+\frac{D^{4 / 5}}{2^{11 / 10} 3} \frac{\xi^{8 / 5} l_{\mathrm{p}}^{2 / 5}}{L} .
$$

By application of (10), the tube diameter is easily obtained as

$$
L_{\perp}=\frac{D^{3 / 5}}{2^{27 / 10}} \frac{\xi^{6 / 5}}{l_{\mathrm{p}}^{1 / 5}}+\frac{D}{2^{5 / 2}} \frac{\xi^{2}}{L} .
$$

Evaluation of the numerical factors holds the following results to first order

$$
L_{\mathrm{d}} \approx 0.66 \xi^{4 / 5} l_{\mathrm{p}}^{1 / 5}, \quad L_{\perp} \approx 0.32 \frac{\xi^{6 / 5}}{l_{\mathrm{p}}^{1 / 5}}
$$

Note that this also determines the confinement free energy of the polymer to $\Delta F \approx 2.14 k_{\mathrm{B}} T \frac{L}{\xi^{4 / 5} l_{\mathrm{p}}^{1 / 5}}$. The leading term of the tube diameter agrees with the established scaling [16]. The additional term's dependence on the inverse polymer length indicates a finite length effect. It can be traced back to the partition sum of a finite polymer (8) and accounts for boundary effects at the end of the tube. If the free energy of infinite polymers (44) is used throughout the calculations, all higher order terms vanish accordingly. In an earlier work [25] another prefactor of $L_{\perp} \approx 0.53 \xi^{6 / 5} l_{\mathrm{p}}^{-1 / 5}$ for the scaling term has been predicted by rather different accounting of obstacles.

It is important to be aware of the subtle difference between the explicit length dependence of the first order term and the implicit dependence on $L$ that enters via the mesh size $\xi=\sqrt{3 / \nu L}$. In a polydisperse polymer solution the $L$ in the mesh size has to be the average polymer length, while the $L$ in the second order terms is the length of the actually observed filament in the tube. In a monodisperse solution as in our theory these quantities are identical.

The importance of the second order term depends heavily on the nature of the polymers making up the network. In Fig. 6] the relative tube width correction obtained by

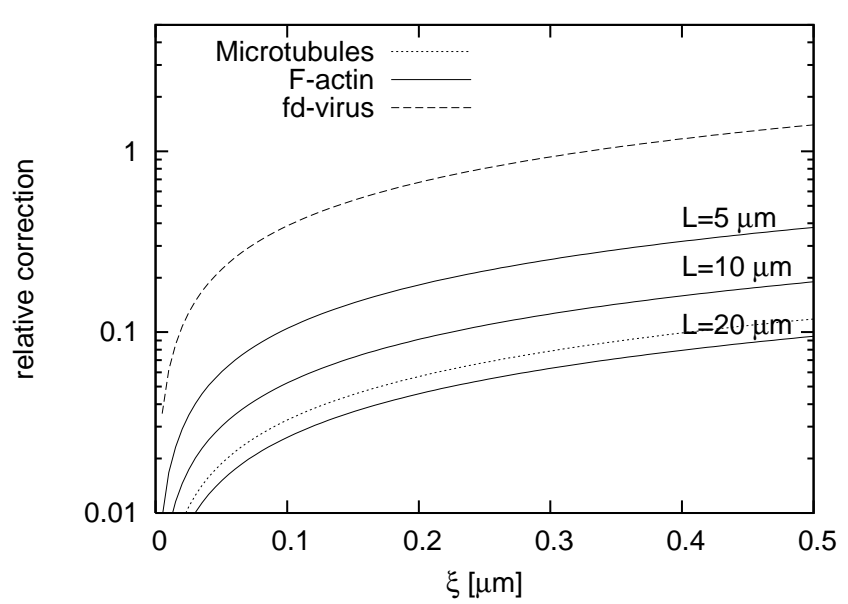

Fig. 6. Relative correction obtained by the second order term of the tube diameter (38) for different biopolymers as a function of mesh size $\xi$. fd-viruses ( $L \approx 0.9 \mu \mathrm{m}, l_{\mathrm{p}} \approx 2.2 \mu \mathrm{m}[26$ ]) show a large correction due to their small length compared to the mesh size, while this effect is rather small in microtubules $\left(L \approx 50 \mu \mathrm{m}, l_{\mathrm{p}} \approx 5000 \mu \mathrm{m}[27,20]\right.$ ). The correction for F-actin has been plotted for different length from a typical length distribution.

the second order term is displayed for several semiflexible biopolymers as a function of mesh size $\xi$. It is interesting to note that the intuitive dependence on the relative persistence length $l_{\mathrm{p}} / L$ present in the second order term of the deflection length is rather negligible. The most dominant effect of the correction term is not obtained for the stiffest biopolymer, a microtubule, but for the small fd-virus. This is due to its small length to mesh size ratio. Finite length effects will influence a large fraction of the polymer strand and not only the boundaries. Given a proper control of polymer length, this effect should be experimentally observable in F-actin solutions.

Focussing back on F-actin, Fig. 7 displays the result of our model in comparison to experimental data [28,29. While theoretical and experimental results are certainly qualitatively comparable, a more detailed discussion is difficult due to the large fluctuations of the measurements. However, it seems reasonable to interpret these measurements regardless of their ambiguity as an upper limit to the tube diameter. Two main reasons cause an experimental observation of tube widths systematically higher than in the presented theory: from a technical point of view the microscope resolution broadens the observed tubes. Additionally, this effect is further enhanced by collective fluctuations of the complete elastic medium that remain unaccounted for in our approach. Contrary, the computer simulations presented below, can be tailored to avoid these effects and study the exact model system used by the theory.

\section{Simulations}

We have conducted intensive numerical simulations of the model system for several reasons: on the one hand they 


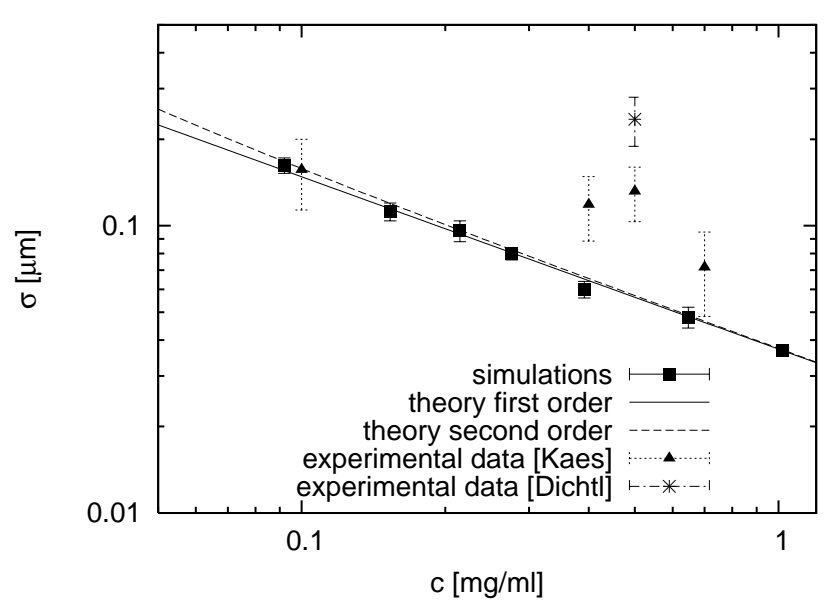

Fig. 7. Comparison of tube diameter from theory, numerical simulations (squares) and reanalyzed experimental measurements (triangles) from [28,29]. While Dichtl has directly measured potential strengths, Käs has recorded the maximal tube width $a$. Therefrom we estimated a lower boundary of $\sigma=a / 6$.

serve as a tool to verify the validity of several approximations used in the theoretical description developed above, being for example the harmonic description of the tube potential or the assumption of a single fluctuation amplitude for the obstacles. Furthermore, the comparison between the simulated transverse fluctuations and the final result of our theory can prove if we have succeeded in correctly predicting the tube diameter in a network of semiflexible polymers. Finally, the simulations give us the chance to analyze observables that go beyond the analytical theory presented. These are in particular distribution functions and open up a further possibility to comparison with experiments.

We use a Monte Carlo simulation of a single polymer in two dimensions that is surrounded by point-like obstacles. This reduction will result in an equal fluctuation amplitude as in the 3D model, because we have assumed the fluctuations along the different coordinates to be independent. Simulating a test polymer in $2 \mathrm{D}$ and measuring its transverse displacement, will thus on average correspond to either $L_{\perp, y}$ or $L_{\perp, z}$ given that the number of obstacle points has been chosen correctly. We calculate this number as the number of stiff rods that cut an arbitrary unit area plane if these rods are of length $L$, density per unit volume $\nu$ and equally distributed both in position and orientation. The approximation as rigid rods is justified by the large persistence length compared to the mesh size. The relation between polymer concentration $\nu$ and pointdensity in simulations $\rho_{\mathrm{MC}}$ then yields (45):

$$
\rho_{\mathrm{MC}}=\frac{2}{\pi} \nu L
$$

Of course, the obstacles will cut the plane under different angles. These can be incorporated via different statistics of the obstacle fluctuations. However, simulations show that no significant differences compared with orthogonal cuts occur. This can be explained by an averaging out of anisotropies in performing ensemble averages. We therefore choose to assume orthogonal intersections of the obstacle polymers with the plane of simulation.

Having defined a suitable conversion for the polymer density, we will proceed to implement the other contribution to the test polymer's Hamiltonian in our simulations, i.e. the persistence length. The test polymer is modelled as a chain of $N$ rigid segments that approximate the continuous contour of a worm-like chain. The joint angle between two segments gives rise to a bending energy summed over all bonds:

$$
\beta H\left(\left\{t_{i}\right\}\right)=k \sum_{i=1}^{N-1} \mathbf{t}_{i} \mathbf{t}_{i+1},
$$

where the $\mathbf{t}_{\mathbf{i}}$ are the tangents and $\mathrm{k}$ is chosen such to reproduce the energy of a semiflexible polymer of persistence length $l_{\mathrm{p}}$. The relation in two dimensions is computed to

$$
\frac{L}{l_{\mathrm{p}}}=-N \ln \left[\frac{I_{1}(k)}{I_{0}(k)}\right]
$$

with $I_{0}$ as modified Bessel function of first kind and its derivative $I_{1}$.

The simulations start from an equilibrium conformation of the test polymer and with obstacle centers $r_{i}^{0}$ that are uniformly distributed. At this point, obstacles are discriminated into those in the half-spaces left and right of the test polymer. During the following evolution of the system, every move of an obstacle and every conformation change of the test polymer is rejected if it would result in a reclassification of any obstacle into the other half-space. Besides this constraint the evolution is only governed by the bending energy of the test polymer and a harmonic potential $U\left(r_{i}\right)=\sigma / 2\left(r_{i}-r_{i}^{0}\right)^{2}$ for every obstacle. During the evolution the transverse displacements $L_{\perp}$ of every bond from the average contour are recorded over the whole evolution. To avoid boundary phenomena, this is only done in the bulk. The whole procedure is then carried out repeatedly for different initial sets of random obstacles and random test polymer conformations. If the computation is repeated for different values of $\sigma$, a function $L_{\perp}(\sigma)$ is obtained from which the point of self consistence $L_{\perp}(\sigma)=\sigma$ and its error can be deduced graphically. Repeating the procedure for different parameters, holds results for the tube diameter in dependence of persistence length $l_{\mathrm{p}}$ and concentration $\nu L$ and can be compared to the theoretical prediction and the available scarce experimental data. As displayed in Fig. 7 the simulation results and the theoretical prediction to both first and second order agree remarkably well. On the basis of the available data any discrimination between first and second order would be bold. However, it has to be considered that any deviations due to lack of simulation time or shortcomings in the Monte Carlo moves will tend to reduce the observed tube width. The obtained simulation results are thus a lower boundary to the real tube diameter.

Even if the good agreement between the theoretical predicted tube diameter and the values observed in numerical simulations suggests our theoretical description to 
be valid, we employ the developed algorithms to explicitly check on some of the assumption made in the course of deriving the tube diameter.

One central assumption in the realm of the tube model is the substitution of an ensemble of neighboring polymers by an effective tube potential. This tube potential is modelled by an harmonic function of strength $\gamma$ as in the Hamiltonian (1). This harmonic assumption seems sensible and is also supported by preliminary experiments with colloidal probes [29]. Our numerical simulations can provide further proof to the exact form of the potential. To this end, we have monitored the transverse displacement as a function of arc length. In the resulting histogram see Fig. 8 (top) for some examples - we identify the distributions maximum as the center position and analyze the form of the potential. Evidently, the resulting profiles in the test polymer's bulk are reasonably Gaussian shaped, while deviations at the boundaries (compare data for $s=0.08$ in Fig. 8 (top)) occur but are negligible for a tube model where $L>L_{\perp}$. For a quantification the ratio of fourth moment to square of second moment of transverse fluctuations

$$
Q=\frac{\overline{(y-\bar{y})^{4}}}{{\overline{(y-\bar{y})^{2}}}^{2}}
$$

was considered. For a perfect Gaussian distribution this quantity evolves to $Q=3$. As shown in Fig. 8 (bottom) this value is also asymptotically obtained in the simulations after sufficient simulation time. These results clearly support the validity of a harmonic tube potential.

In contrast to the classical picture of an Edwards-tube with a rather homogeneous diameter the simulations reveal a rather large variability in the local tube diameter as has also been observed experimentally [28,29]. Carrying out extensive simulations in a large number of different obstacle environments allows one to record the distribution function of the tube diameters. This is of crucial importance, as our theoretical description has assumed that the tube diameter - and hence due to self-consistency also the obstacle fluctuation width - can be described by a single characteristic value. This approach only seems feasible if the distribution described by the characteristic value is reasonably well peaked. The simulations prove that the resulting distribution is indeed equipped with a well-defined peak (Fig. 9). However, the variability of the observed tube diameters is rather large with a half-width of the size of the average tube diameter itself. We observe a sharp cut-off for small tube widths while the distribution's tail to wide tubes is longer. The behavior at small tube width is dominated by the energy cost of confining a polymer into an increasingly smaller tube and can thus be considered as a polymer property. On the contrary the distribution at tube widths larger than the average diameter is due to void spaces. These will follow an exponential decay and are therefore a characteristic of the network architecture.

Finally, the numerical simulations provide a means to explicitly check if the self-consistence is guaranteed in spite of the simplifying assumption of a single fluctuation
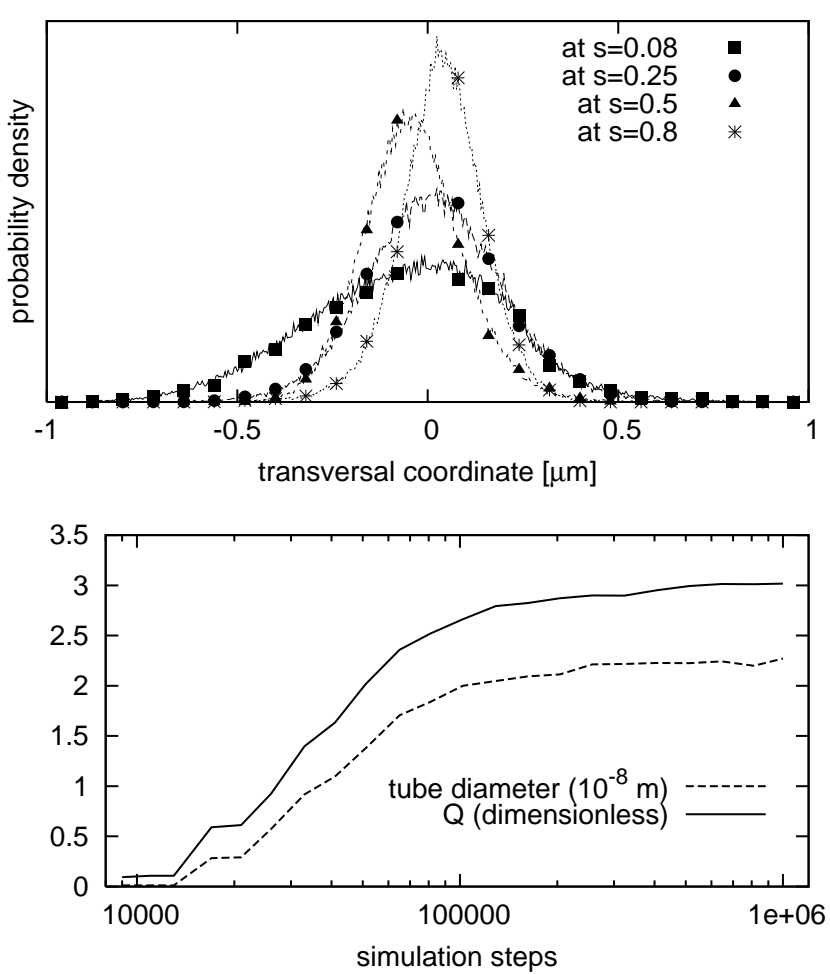

Fig. 8. (top) Distribution of transverse excursions at different arc-lengths $s$ shows a Gaussian potential profile with rather large variability in the potential width. At the test polymer's boundaries deviations occur. (bottom) After sufficient simulation time the ratio $Q$ (solid) approaches the characteristic value $Q=3$ of a Gaussian distribution. The transverse fluctuation area (dashed) converges likewise.

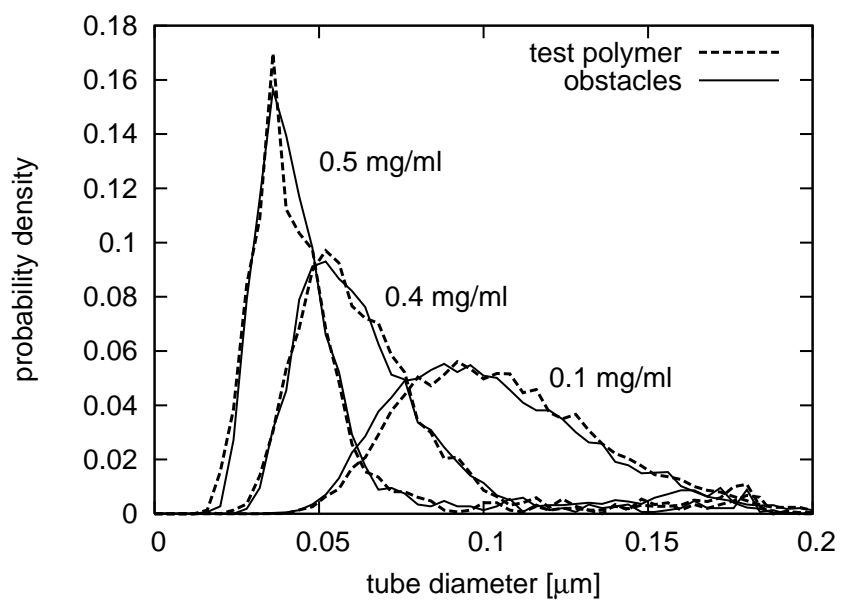

Fig. 9. Distribution of $L_{\perp}$ sampled over polymer arc-length and different obstacle environments. Distributions are wellpeaked and exhibit longer tails at large tube widths. The noise at diameters far from the distributions maximum is an artefact from the numerical discretization. 
width. To this end, we have used the resulting histogram of tube diameters from above to compute a normalized distribution function. The fluctuation width of the obstacles are now initialized according to this very distribution. The resulting histogram of tube diameters is then again fed back into the simulation as obstacle fluctuation distribution. This procedure is carried out until both distributions converge against each other in a self-consistent manner. Surprisingly, this is already the case after the first iteration step of the process as displayed in Fig. 9. This gives strong evidence that due to the self-averaging over obstacles the modelling of a network with Gaussian tube profile and a single average tube diameter is sufficient to describe the physical reality.

\section{Conclusion}

We have presented a new approach to determine the absolute value of the tube diameter in semiflexible polymer networks supported by computer simulations. To this end the deflection length of a polymer in a hypothetical harmonic tube was connected to the tube's diameter via the free energy cost for finite length polymers. The assumption of a harmonic tube was confirmed by simulation results. By decomposition into independent stiff rods of appropriate length, we were able to establish an implicit equation for the deflection length. The resulting tube width $L_{\perp}$ is in agreement with the established scaling law $L_{\perp}=c \xi^{6 / 5} l_{\mathrm{p}}^{-1 / 5}$ with mesh size $\xi=\sqrt{3 / \nu L}$ and persistence length $l_{\mathrm{p}}$. Our theory provides a prefactor of $c \approx 0.32$ and a higher order term that accounts for finite length effects and scales with $\xi^{2} / L$.

The available experimental data is consistent with our predictions. However, its quality does not allow for detailed comparison. To provide a precise validation, we have complemented our theoretical work by extensive Monte Carlo simulations of a test polymer in an environment of obstacles. The resulting self-consistent tube widths perfectly match the theoretical value predicted. This strongly supports the validity of the absolute value for the concentration dependent tube diameter.

Furthermore, we have employed simulations to observe properties beyond the analytical theory. We have recorded the distribution function of tube widths in a network for different concentrations. Thereby we were able to explicitly confirm self-consistency of the simplifying model with a fixed tube diameter.

Both our theoretical predictions, e.g. the finite length contributions to the tube diameter, and our simulation data, e.g. the distribution functions, provide the opportunity of feasible experimental comparison. On the theoretical side, the significance of correlations and collective fluctuations of the complete medium, as well as an analytical model of distribution functions may open up promising continuations of this work.

We kindly acknowledge helpful discussions with M. Degawa, M. Giesen, R. Merkel and M. Romanoska. Financial support of the German Excellence Initiative via the program "Nanosystems Initiative Munich (NIM)" is gratefully acknowledged. HH acknowledges support by the international graduate program Nano-Bio-Technology funded by the Elite Network of Bavaria.

\section{A Rigid Rod Statistics I}

To relate the polymer concentration of a network to the obstacle density per unit area in the simulation, we calculate the number of randomly distributed and oriented stiff rods per unit volume that intersect with a unit plane. Every intersecting rod will be described by its polar and azimuth angle relative to the unit plane, the point of intersection and the distance between center of mass and intersection point. Because of rotational symmetry the problem is independent of the azimuth angle and because of uniform density it is independent of one of the coordinates of the intersection point. Hence, the problem is equivalent in two dimensions to the number of rods per unit area that intersect a unit line (see Fig. 10](left)). The plane contains $\nu$ rigid rods per unit area with random orientation $\alpha$ and center of mass position. The number of intersections $\rho_{\mathrm{MC}}$ with the unit line (bold dashed) is computed by parameterizing the center of mass $(\mathrm{C})$ by the coordinate $z$ of the intersection point $(\mathrm{P})$ with the unit line, the distance $s$ between $\mathrm{C}$ and $\mathrm{P}$ and the angle $\alpha . \rho_{\mathrm{MC}}$ is then obtained as the integral over all possibly intersecting rods:

$$
\rho_{\mathrm{MC}}=\frac{2 \nu}{2 \pi} \int d r^{2} \int_{0}^{\pi} d \alpha
$$

where the factor 2 accounts for the fact that any rod configuration can be realized by two angles $\alpha$ since the rods have no direction. The integration area has to be chosen appropriately to only include intersecting rods. As $r=(\sin (\alpha) s, x-\cos (\alpha) s)$ the Jacobian determinant of the coordinate transformation to integration variables is $\partial r / \partial(x, s)=\sin (\alpha)$ and the integral evolves to

$$
\rho_{\mathrm{MC}}=\frac{\nu}{\pi} \int_{-1 / 2}^{1 / 2} d x \int_{-L / 2}^{L / 2} d s \int_{0}^{\pi} \sin (\alpha)=\frac{2}{\pi} \nu L .
$$

\section{B Rigid Rod Statistics II}

We derive the radial density of obstacles that effectively hinder the test rods fluctuations. To this end we consider the test rod to be aligned along the z-axis without loss of generality (see Fig. 10 (right)). As criterion for effective obstruction of transverse fluctuations between test rod and obstacle, we demand that the line connecting their points of closest approach $(\overline{P O})$ is orthogonal to both polymers. As a projection of the obstacle to the plane spanned by the test rod and $\overline{P O}_{\perp}$ (dashed) recovers the setup discussed in Appendix $\mathrm{A}$, the coordinates of the center of mass $(\mathrm{C})$ can readily be extended to three dimensions by the radial distance $R$ and the angle $\beta$ to $r=(\sin (\alpha) s \sin (\beta)-\cos (\beta) R, \sin (\alpha) s \cos (\beta)+$ 
$\sin (\alpha) R, x-\cos (\alpha) s)$ and with the Jacobian determinant $|\partial r / \partial(x, s, R)|=\sin (\alpha)$ the integration gives

$$
\begin{aligned}
\rho(R) & =\frac{2 \nu}{4 \pi} \int_{-\frac{L}{2}}^{\frac{L}{2}} d x \int_{-L / 2}^{L / 2} d s \int_{0}^{\pi} d \alpha \int_{0}^{2 \pi} d \beta \sin ^{2}(\alpha) \\
& =\frac{\pi}{2} \nu L^{2} .
\end{aligned}
$$

Consequently, the density seen by an stiff segment of length $\bar{L}$ in the IRM will be

$$
\rho=\frac{\bar{L}}{L} \frac{\pi}{2} \nu L^{2}=\frac{\pi}{2} \nu L \bar{L}
$$

Note that in the simplified geometry the obstacle density is not a complete radial density but a line density of obstacles on one of the four axes (positive and negative $y$ and $z$ axis). To recover the complete radial density, one has to sum over all of these. Hence the obstacle density on either one of the four axes has to be:

$$
\rho=\frac{\pi}{2} \frac{\nu L \bar{L}}{4} .
$$
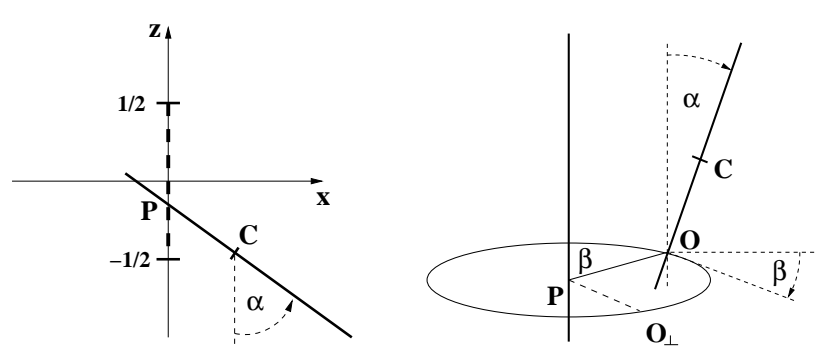

Fig. 10. (left) Sketch of a rod with center of mass (C) and orientation $\alpha$ intersecting a arbitrary unit line (dashed) to illustrate the calculation of the number of intersecting rods $\rho_{\mathrm{MC}}$. (right) Two rods with minimal distance $R$ are considered to be mutually interacting only if their line of closest approach $(\overline{O P})$ is orthogonal to both. $O_{\perp}$ only serves to illustrate the analogy to the two-dimensional setup (see text).

\section{Mode analysis of polymer and tube}

Using the dimensionless arc-length $\tilde{s}=s / L$, polymer conformation $\tilde{y}(\tilde{s})=y(\tilde{s} L) / L$, tube center $\tilde{y}^{0}(\tilde{s})=y^{0}(\tilde{s} L) / L$ and persistence length $\eta=l_{\mathrm{p}} / L$ the Hamiltonian (1) can be written for one transverse coordinate as

$$
\beta H=\frac{\eta}{2} \int_{0}^{1} d \tilde{s}\left[\left(\partial_{\tilde{s}}^{2} \tilde{y}\right)^{2}+l_{\mathrm{d}}^{-4}\left(\tilde{y}-\tilde{y}^{0}\right)^{2}\right] .
$$

For free boundary conditions the Hamiltonian is diagonalized by an orthonormal set of eigenfunctions $\psi_{k} \approx$ $\sin \left(q_{k} \sigma+\phi_{k}\right)$ with $q_{k} \approx \pi(k+1 / 2)$ [30. Expanding both polymer and tube center in modes as $\tilde{y}(\sigma)=\sum_{k} y_{k} \psi_{k}$ and $\tilde{y}^{0}(\sigma)=\sum_{k} y_{k}^{0} \psi_{k}$ and using the orthogonality of the eigenfunctions allows one to write the Hamiltonian in the suggestive form

$$
\begin{aligned}
\beta H= & \frac{\eta}{2} \sum_{k}\left(q_{k}^{4}+l_{\mathrm{d}}^{-4}\right)\left(y_{k}-\frac{y_{k}^{0}}{1+\left(q_{k} l_{\mathrm{d}}\right)^{4}}\right)^{2} \\
& +\frac{q_{k}^{4} y_{k}^{0}}{1+\left(q_{k} l_{\mathrm{d}}\right)^{4}} .
\end{aligned}
$$

By comparison to (49) one can read of the minimum of the confinement potential, i.e. the average tube center:

$$
\overline{y_{k}}=y_{k}^{0} /\left(1+q_{k}^{4} l_{\mathrm{d}}^{4}\right) .
$$

Additionally, this allows to write the complete transverse fluctuations as a sum over the inverse confinement strength of all modes:

$$
L_{\perp}^{2}:=\frac{1}{L} \int_{0}^{L} d s \overline{(x(s)-\overline{x(s)})^{2}}=\frac{L^{2}}{\eta} \sum_{k} \frac{1}{q_{k}^{4}+l_{\mathrm{d}}^{-4}}
$$

For the dimensionless function $h\left(l_{\mathrm{d}}\right)$ relating the tube diameter to the deflection length follows:

$$
h(l d)=\sum_{k} \frac{1}{q_{k}^{4}+l_{\mathrm{d}}^{-4}} .
$$

\section{References}

1. J. Howard. Mechanics of Motor Proteins and the Cytoskeleton. Sinauer Associates, Sunderland, 2001.

2. A. R. Bausch and K. Kroy. Nature Phys., 2:231, 2006.

3. D. Pantaloni, C. L. Clainche, and M. F. Carlier. Science, 292:1502, 2001.

4. M. L. Gardel, J. H. Shin, F. C. MacKintosh, L. Mahadevan, P. Matsudaira, and D. A. Weitz. Science, 304:1301, 2004.

5. B. Wagner, R. Tharmann, I. Haase, M. Fischer, and A. R. Bausch. Proc. Nat. Acad. Sci., 103:13974, 2006.

6. B. Hinner, M. Tempel, E. Sackmann, K. Kroy, and E. Frey. Phys. Rev. Lett., 81:2614, 1998.

7. T. T. Perkins, D. E. Smith, and S. Chu. Science, 264:819, 1994.

8. J. Käs, H. Strey, and E. Sackmann. Nature, 368:226, 94.

9. P. G. de Gennes. Scaling Concepts in Polymer Physics. Cornell University Press, Ithaca, NY, 1979.

10. M. Doi and S. F. Edwards. The Theory of Polymer Dynamics. Clarendon Press, Oxford, 1986.

11. T. Odijk. Macromolecules, 16:1340, 1983.

12. F. C. MacKintosh, J. Käs, and P. A. Janmey. Phys. Rev. Lett., 75:4425, 1995.

13. H. Isambert and A. C. Maggs. Macromolecules, 29:1036, 1996.

14. R. Everaers, S. K. Sukumaran, G. S. Grest, C. Svaneborg, A. Sivasubramanian, and K. Kremer. Science, 303:823, 2004.

15. C. Tzoumanekas and D. N. Theodorou. Macromolecules, 39:4592, 2006.

16. A. N. Semenov. J. Chem. Soc. Faraday. Trans., 82:317, 1986. 
17. O. Kratky and G. Porod. Rec. Trav. Chim., 68:1106, 1949.

18. N. Saito, K. Takahashi, and Y. Yunoki. J. Phys. Soc. Jpn., 22:219, 1967.

19. S. Kaufmann, J. Ks, W. H. Goldmann, and G. Isenberg. FEBS Lett., 314:203, 1992.

20. F. Gittes, B. Mickey, J. Nettleton, and J. Howard. J. Cell Biol., 120:923, 1993.

21. L. Le Goff, O. Hallatschek, E. Frey, and F. Amblard. Phys. Rev. Lett., 89:258101, 2002.

22. C. F. Schmidt, M. Baermann, Gerhard Isenberg, and Erich Sackmann. Macromolecules, 22:3638, 1989.

23. T. Burkhardt. J. Phys. A., 28:629, 1995.

24. H. Kleinert. J. Math. Phys., 27:3003, 1986.

25. D. C. Morse. Phys. Rev. E, 63:031502, 2001.

26. F. G. Schmidt, B. Hinner, E. Sackmann, and J. X. Tang. Phys. Rev. E, 62:5509, 2000.

27. F. Pampaloni, G. Lattanzi, A. Jonas, T. Surrey, E. Frey, and E.-L. Florin. Proc. Nat. Acad. Sci., 103:10248, 2006.

28. J. Käs, H. Strey, J. X. Tang, D. Finger, R. Ezzell, E. Sackmannt, and P. A. Janmey. Biophys. J., 70:609, 1996.

29. M. A. Dichtl and E. Sackmann. New. J. Phys., 1:18.1, 1999.

30. S. Aragon and R. Pecora. Macromolecules, 18:1868, 1985. 